\title{
Drenos torácicos mal posicionados diagnosticados por exame de imagem
}

\author{
Malor positioned thoracic drains diagnosed by image examination \\ Drenajes torácicos en posiciones malas diagnosticados mediante examen de imagen
}

\section{Resumo}

A drenagem torácica é um método que através no qual através de um dreno é retirado substâncias seja ela sanguinolentas como em um hemotórax, ou o ar, no caso do pneumotórax, para que assim seja possível o deslizamento entre as pleuras e mantenha uma adequada expansão pulmonar. No caso de pacientes em que tiveram má inserção do dreno na cavidade pleural pode haver uma piora do quadro clínico, com uma redução da ventilação, insuficiência respiratória, ocorrência de infeções, ou mesmo o pneumotórax iatrogênico. Objetivo: Analisar os achados de imagem de drenos de tórax mal posicionados na tomografia computadorizada. Materiais e métodos: Esta pesquisa trata-se de um estudo retrospectivo, quantitativo com enfoque descritivo, no qual foi utilizado dados de exames de tomografia computadorizadas disponibilizados pela clínica Polimagem Radiodiagnóstico no município de Marabá-PA. Resultados: No presente estudo o grupo que tem maior incidência de complicações relacionadas ao posicionamento inadequado do dreno foi do sexo masculino, entre 19 a 59 anos, indicado por causas traumáticas, o local mais comum de inserção do dreno incorreto ocorreu na região intraparenquimatosa causando como principais complicações o pneumotórax, sangramentos e nos casos mais avançados o surgimento de fistulas. Conclusão: Os achados do exame de imagem tiveram essencial importância para detectar a iatrogenia causada pelos drenos mal inseridos, os resultados devem ser ampliado para evitar a inadequada colocação de dreno, adotando também protocolos hospitalares padronizados e treinamento da equipe de saúde para sua utilização.

Palavras-chave: Drenagem torácica; Trauma torácico; Tomografia computadorizada; Complicações.

\begin{abstract}
Thoracic drainage is a method whereby substances are removed through a drain, whether bloody as in a hemothorax, or air, in the case of pneumothorax, so that it is possible to slide between the pleura and maintain an adequate pulmonary expansion. In the case of patients who have had poor insertion of the drain into the pleural cavity, there may be a worsening of the clinical picture, with reduced ventilation, respiratory failure, occurrence of infections, or even iatrogenic pneumothorax. Objective: To analyze the imaging findings of poorly positioned chest drains on computed tomography. Materials and methods: This research is a retrospective, quantitative study with a descriptive focus, in which data from computed tomography exams provided by the Polimagem Radiodiagnóstico clinic in the municipality of Marabá-PA were used. Results: In the present study, the group with the highest incidence of complications related to inadequate drain placement was male, between 19 and 59 years old, indicated by traumatic causes, the most common location for the insertion of the incorrect drain occurred in the intraparenchymal region causing main complications are pneumothorax, bleeding and in more advanced cases the appearance of fistulas. Conclusion: The findings of the imaging examination were essential for detecting iatrogenesis caused by poorly
\end{abstract}


inserted drains, the results should be expanded to avoid inadequate drain placement, also adopting standardized hospital protocols and training of the health team to use them.

Keywords: Thoracic drainage; Thoracic trauma; Computed tomography; Complications.

\section{Resumen}

El drenaje torácico es un método mediante el cual se extraen sustancias a través de un drenaje, ya sea sanguinolento como en un hemotórax, o aéreo, en el caso de un neumotórax, de manera que se pueda deslizar entre la pleura y mantener una adecuada expansión pulmonar. En el caso de pacientes que han tenido mala inserción del drenaje en la cavidad pleural, puede haber un empeoramiento del cuadro clínico, con ventilación reducida, insuficiencia respiratoria, aparición de infecciones o incluso neumotórax iatrogénico. Objetivo: Analizar los hallazgos de imagen de drenajes torácicos mal posicionados en tomografía computarizada. Materiales y métodos: Esta investigación es un estudio retrospectivo, cuantitativo con enfoque descriptivo, en el que se utilizaron datos de exámenes de tomografía computarizada proporcionados por la clínica de Radiodiagnóstico Polimagem del municipio de Marabá-PA. Resultados: En el presente estudio, el grupo con mayor incidencia de complicaciones relacionadas con la colocación inadecuada del drenaje fue el masculino, entre 19 y 59 años, indicado por causas traumáticas, la ubicación más común para la inserción del drenaje incorrecto ocurrió en el intraparenquimatoso La región que origina las principales complicaciones son el neumotórax, el sangrado y en casos más avanzados la aparición de fístulas. Conclusión: Los hallazgos de la exploración por imágenes fueron fundamentales para detectar iatrogénesis por drenajes mal insertados, los resultados deben ampliarse para evitar la colocación inadecuada de los drenajes, adoptando también protocolos hospitalarios estandarizados y capacitando al equipo de salud para su uso.

Palabras clave: Drenaje torácico; Traumatismo torácico; Tomografía computarizada; Complicaciones.

\section{Introdução}

A drenagem pleural é um procedimento médico no qual há a colocação de tubo ou cateter através da parede torácica na cavidade pleural. É comumente utilizado para drenar ar ou fluidos, tendo por finalidade a recuperação da expansão pulmonar e evitando complicações secundárias, podendo ser uma drenagem apical ou basal nas pleuras mediastinais (Huggins et al, 2019).

$\mathrm{Na}$ fisiologia pulmonar, deve haver um equilíbrio na cavidade pleural para manter constante a concentração protéica do fluído pleural. Esse equilíbrio é garantido pelos movimentos de expiração e inspiração, os quais são responsáveis por auxiliar na reabsorção dos líquidos, partículas, e na progressão pelos vasos linfáticos. Para ocorrer essa movimentação, deve sempre haver um gradiente de pressão negativa, o que faz que mesmo em repouso os pulmões permaneçam expandidos (Cipriano; Dessote, 2011).

A drenagem torácica tem como finalidade também além da remoção de substâncias para possibilitar a reexpansão pulmonar, pode criar uma via de acesso para uma pleurodese, que consiste na utilização de substâncias, como o talco, que é capaz de criar aderências entre as pleuras parietais e a visceral, e assim evitar a recorrência de um derrame pleural (Araújo, 2017).

Diversos fatores, por exemplo, atos cirúrgicos, doenças pleurais ou traumas, podem causar um desarranjo desse sistema, provocando acúmulo de fluidos no espaço pleural, e dessa forma, prejudicar a mecânica pulmonar fisiológica. Essa situação altera os níveis pressóricos do sistema pulmonar, podendo levar ao colapso dos pulmões e insuficiência respiratória de intensidade variável (Cipriano; Dessote, 2011).

A utilização das técnicas de drenagem remonta à época de Hipócrates, na Grécia antiga, que no século V a.C. realizava drenagem de empiemas pleurais com tubos e cânulas. Até o século XIX, era recomendado a técnica de drenagem torácica aberta com mortalidade de até $70 \%$ dos casos. A partir do século XX, adotou-se a drenagem por tubo fechado através das recomendações de Comissões, reduzindo a mortalidade para 4,3\% (Davies et al, 2010).

Desde então, a drenagem com circuito fechado é a técnica padrão. Ela dispõe de dispositivos de material maleável de tubos de silicone e frascos graduados com selo d'água, o que possibilita maior conforto ao paciente e facilidade no procedimento, além de ser possível contabilizar o débito da drenagem (Mendes; Hirano, 2018). 
Atualmente, o procedimento é largamente empregado na prática médica diária e considerado como ato relativamente simples, sendo feito à beira do leito, no centro cirúrgico, ou na emergência. Os profissionais mais indicados para a realização da técnica seriam os cirurgiões torácicos, porém, na maioria dos hospitais também é feito por médicos emergencistas, intensivistas, pneumologistas, e radiologistas intervencionistas. Além da instalação do dispositivo, seu controle também é frequentemente atribuição de não especialistas em cirurgia torácica. Dessa forma, os tipos de cuidados costumam variar muito, podendo afetar o resultado do procedimento (Cipriano; Dessote, 2011).

Devido a estes fatores, apesar da drenagem torácica ser uma técnica simples e trazer muitos benefícios aos pacientes, ela nem sempre é um procedimento inofensivo, pode determinar grande potencial de morbidade e mortalidade. As complicações da técnica podem ser resultantes de desconhecimento da anatomia torácica, treinamento inadequado, ou falta de supervisão de profissionais pouco experientes ou ainda em treinamento (Medeiros, 2019).

A realização da toracocentese está intimamente ligada com a ocorrência iatrogênica de pneumotórax, portanto, é indicada que seja realizada uma ultrassonografia antes do procedimento, que garante que a drenagem seja realizada com maior segurança, podendo evitar o pneumotórax em até $90 \%$ dos casos, bem como a possibilidade de retirada de maior quantidade líquido, que é mais eficiente quando se utiliza a ultrassonografia auxiliar nesses pacientes (Perazzo, 2013).

Estudos relataram a incidência de $3 \%$ de complicações precoces, como mal posicionamento do dreno ou pneumotórax, e $8 \%$ de complicações tardias, como deslocamento do tubo e infecções. Além disso, também foram relatadas consequências desastrosas devido a inserção do tubo incorreta como: perfuração do ventrículo direito ou esquerdo, da artéria pulmonar principal e até do esôfago (Filosso et al, 2017).

O mal posicionamento resulta em drenagem inadequada de ar levando o paciente a maior risco de morte. Muitas vezes, o diagnóstico de uma posição anômala do tubo é difícil, pois as manifestações clínicas podem estar ausentes. Para auxílio diagnóstico, a radiografia de tórax é rotineiramente obtida em pacientes após uma drenagem para avaliar a posição e monitorização pleural, bem como a reexpansão pulmonar. Porém, estudos comprovam que não é a modalidade mais confiável para detectar os deslocamentos do dreno (Lim et al, 2005).

Atualmente, a tomografia computadorizada (TC) é considerada a melhor ferramenta de diagnóstico por imagem para avaliação após a inserção de um dreno. É consideravelmente disponível nos serviços de saúde, rápida, e capaz de fornecer informações inatingíveis com exames radiográficos (Lim et al, 2005). Nesse contexto, o presente estudo visa analisar os achados de imagem de drenos mal posicionados diagnosticados a partir de exames de tomografia de tórax, devido a importância da identificação dessa situação na prática clínica para diminuir as chances de complicações decorrentes do procedimento.

Medidas podem ser implementadas para evitar complicações após colocação de drenos torácicos, tais como o acompanhamento com a fisioterapia respiratória, que tem como finalidade o aumento da expansão da complacência pulmonar, o uso de analgesia adequada, além do estabelecimento de protocolos específicos, que visam reduzir as complicações nas vítimas de traumatismo que necessitam da drenagem de tórax. Quando o traumatismo compromete outras regiões do corpo, isso passa a afetar negativamente a evolução, visto que cerca de $19 \%$ dos pacientes podem desenvolver empiema por esse motivo, sendo que nesses casos é necessária laparotomia exploratória seguida do procedimento de drenagem torácica nesses pacientes (Abreu, 2015).

\section{Objetivo}

\subsection{Geral}

Analisar os achados de imagem de drenos de tórax mal posicionados na tomografia computadorizada. 


\subsection{Específicos}

Caracterizar o perfil geral dos pacientes com idade, gênero e as causas da indicação clínica para a drenagem torácica. Descrever o local do posicionamento incorreto do dreno torácico.

Discorrer sobre principais complicações clínicas do mal posicionamento encontrados nos exames de imagem.

\section{Metodologia}

\subsection{Aspectos éticos}

Os dados dos participantes desta pesquisa foram estudados segundo os preceitos da declaração de Helsink e do Código de Nuremberg, respeitadas as Normas de Pesquisa Envolvendo Seres Humanos (Res. CNS 466/12) do Conselho Nacional de Saúde. O estudo foi previamente aprovado pelo parecer $\mathrm{n}^{\mathrm{o}}$ : 3.967 .391 do comitê de ética em pesquisa da Universidade Estadual do Pará.

\subsection{Amostra}

Esta pesquisa trata-se de um estudo retrospectivo, quantitativo com enfoque descritivo, no qual foi utilizado dados de exames de imagem tomográficos de 24 pacientes obtidos da clínica Polimagem Radiodiagnósticos após a assinatura de um termo de consentimento da utilização de dados TCUD, os pacientes do estudo foram oriundos do Hospital Municipal de Marabá e os exames foram realizados no período 22 de outubro de 2018 até o dia 31 de março de 2020.

\subsection{Critérios de inclusão e exclusão}

Os critérios de inclusão foram os pacientes que tiveram indicação para drenagem torácica e apresentaram alterações na inserção do dreno visualizados em exames de tomografia computadorizada, ser do sexo masculino ou feminino e ter idade entre 0 e maior ou igual a 80 anos.

Os critérios de exclusão foram os pacientes que realizaram a colocação do dreno, porém com inserção correta, sem alterações visualizadas na tomografia computadorizada.

\subsection{Local de estudo}

Foi realizado no município Marabá localizado ao sul da capital do Estado (Belém), distando destes cerca de 485 quilômetros, ocupando uma área de 15.128,058 quilômetros, com uma população estimada em 275.086 habitantes para 2018 (IBGE, 2010), nas dependências da Clínica Polimagem Radiodiagnósticos, localizado na avenida Nagib Mutran 433, núcleo cidade nova, que recebe encaminhamentos de pacientes que se encontram internados no Hospital Municipal de Marabá, por conta de contrato celebrado com o poder público.

\subsection{Instrumentos}

\subsubsection{Coleta de exames tomográficos}

Foram coletados após a assinatura do termo de consentimento da utilização de dados da clínica Polimagem radiodiagnósticos 24 exames tomográficos nos quais foram verificadas a idade, o gênero, a indicação da drenagem torácica e as alterações na localização dos drenos, isto é, onde que se encontram fora do espaço pleural.

\subsubsection{Organização dos dados}

Os dados foram organizados de acordo com a idade, gênero dos pacientes, indicação e local de maior prevalência de inserção inadequada dos drenos demostrados na tomografia computadorizada. 


\subsection{Análise de dados}

Após a coleta dos dados, as informações demográficas foram organizadas e comparadas em planilhas do programa Microsoft Excel $2019^{\circledR}$, sendo utilizado representações gráficas por meio de tabelas e gráficos. Além disso, foram analisados os locais mais frequentes de inserção inadequado do dreno na cavidade torácica com base nos exames tomográficos dos 24 pacientes com posicionamento inadequado do dreno na cavidade torácica no período 22 de outubro de 2018 até o dia 31 de março de 2020.

\subsection{Riscos e benefícios.}

Os riscos do presente estudo é o de exposição de dados dos pacientes, porém esse risco será evitado pela garantia de que os dados só serão utilizados para fins científicos, e os exames identificados apenas por números sem envolver o nome real do paciente. Além do acesso a essas informações serem exclusivas aos pesquisadores envolvidos na pesquisa, somente será utilizada pelo tempo necessário à conclusão do estudo.

Os benefícios dessa pesquisa é a possibilidade de adquirir novos conhecimentos sobre a inserção inadequada dos drenos torácicos, e principalmente na utilização de novos meios de utilizar exames de imagem como no caso de ultrassonografia, o qual facilitará o aperfeiçoamento da formação dos profissionais da área de saúde para o manejo adequado do paciente, evitando está complicação para pacientes futuros.

Além disso, a comunidade cientifica irá se beneficiar com informações sobre a faixa etária e local mais acometido com a inserção inadequada de drenos torácicos, possibilitando dessa forma, o enriquecimento da produção cientifica e aprofundamento em estudos futuros sobre as complicações clínicas envolvidas nessas situações.

\section{Resultados}

Foram confirmados cerca de 24 pacientes atendidos no Hospital Municipal de Marabá que tiveram mal posicionamento do dreno torácico dentre os anos de 2018 e 2020. Quando comparamos a série histórica percebe-se no ano de 2018 houve um menor número de casos de posicionamento inadequado de drenos torácicos, em comparação com os anos de 2019 e de 2020, o qual correspondeu a 16,66\% (n=4). Já no ano de 2019, verificou-se uma maior incidência de inserção inadequada do dreno, o qual corresponde cerca de 54,16\% (n=13) dos procedimentos, conforme o Gráfico 1.

Gráfico 1. Número de mal inserção de drenos torácicos por anos.

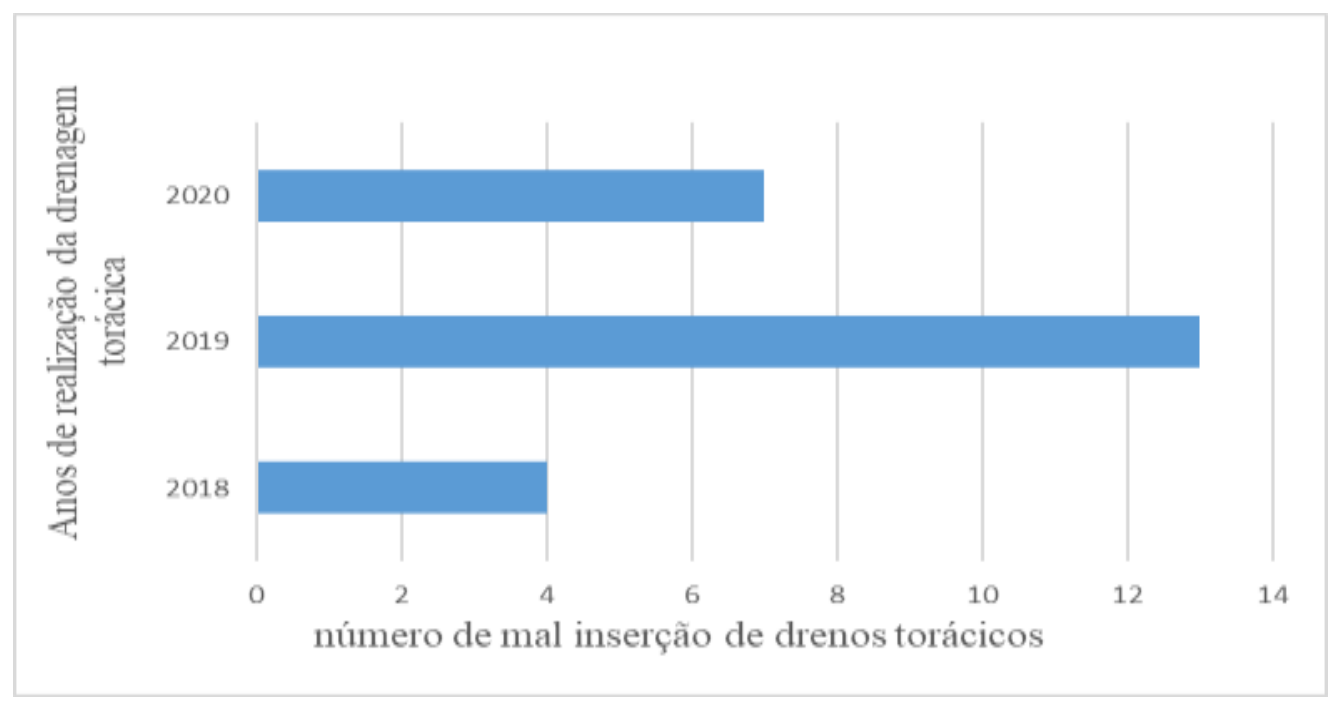

Fonte: Dados da pesquisa. 
Research, Society and Development, v. 10, n. 4, e27410414135, 2021

(CC BY 4.0) | ISSN 2525-3409 | DOI: http://dx.doi.org/10.33448/rsd-v10i4.14135

Gráfico 2. Faixa etária dos pacientes submetidos a drenagem de tórax inadequada.

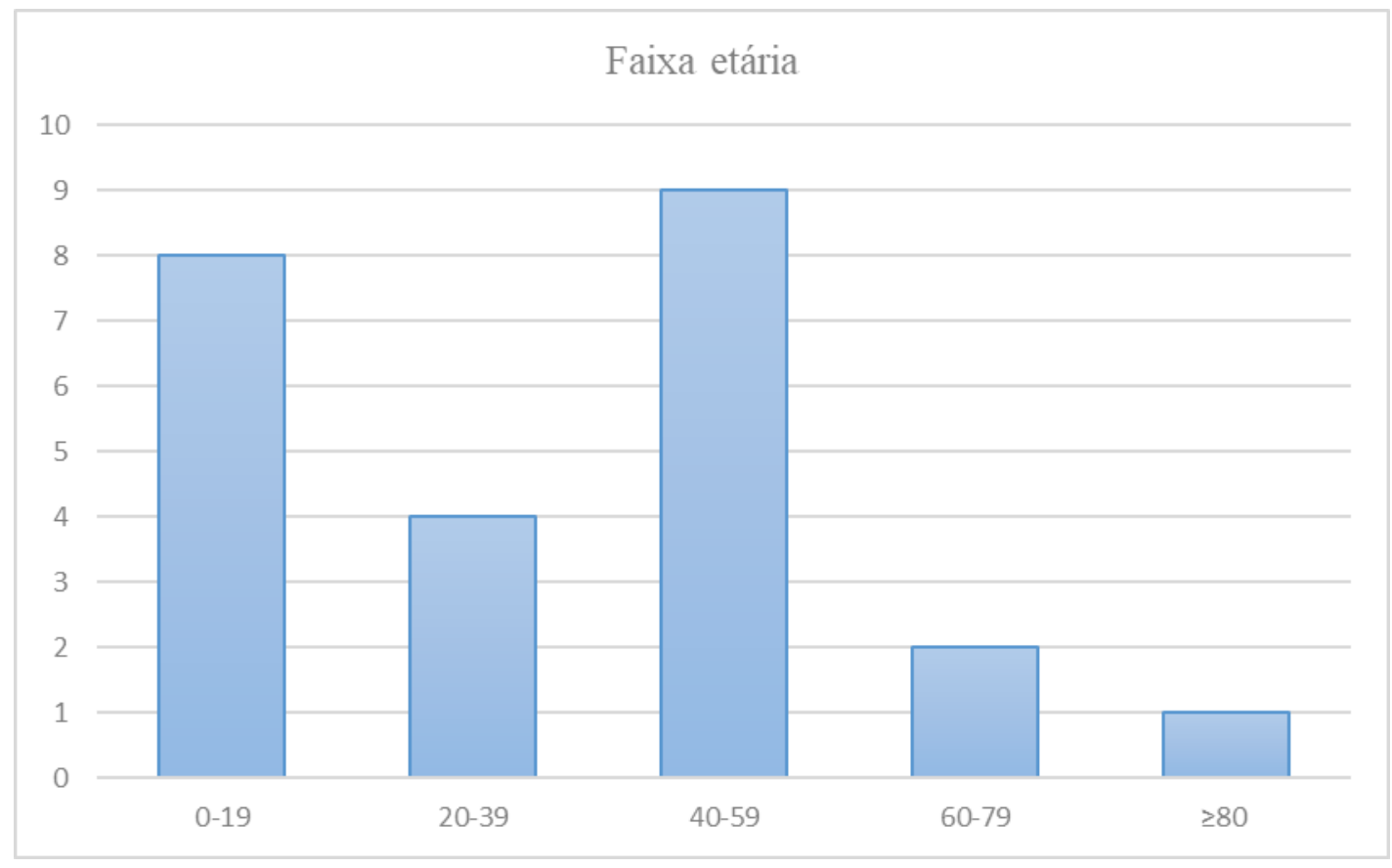

Fonte: Dados da pesquisa.

O Gráfico 2 mostra a faixa etária em que ocorreram a inserção inadequada de drenos torácicos, observando-se que a faixa etária de maior prevalência de ocorrência foi entre 40-59 anos o qual corresponde cerca de 37,5\% (n=9), sendo a segunda maior observada foi entre 0-19 anos correspondente a 33,33\%.

A faixa etária de menor prevalência de drenagem torácica foram os pacientes maiores que 80 anos, o qual correspondeu apenas a 4,16\% dos pacientes, verificando um menor número de idoso submetidos a drenagem torácica.

Gráfico 3. Gêneros dos pacientes com inserção inadequada do dreno torácico.

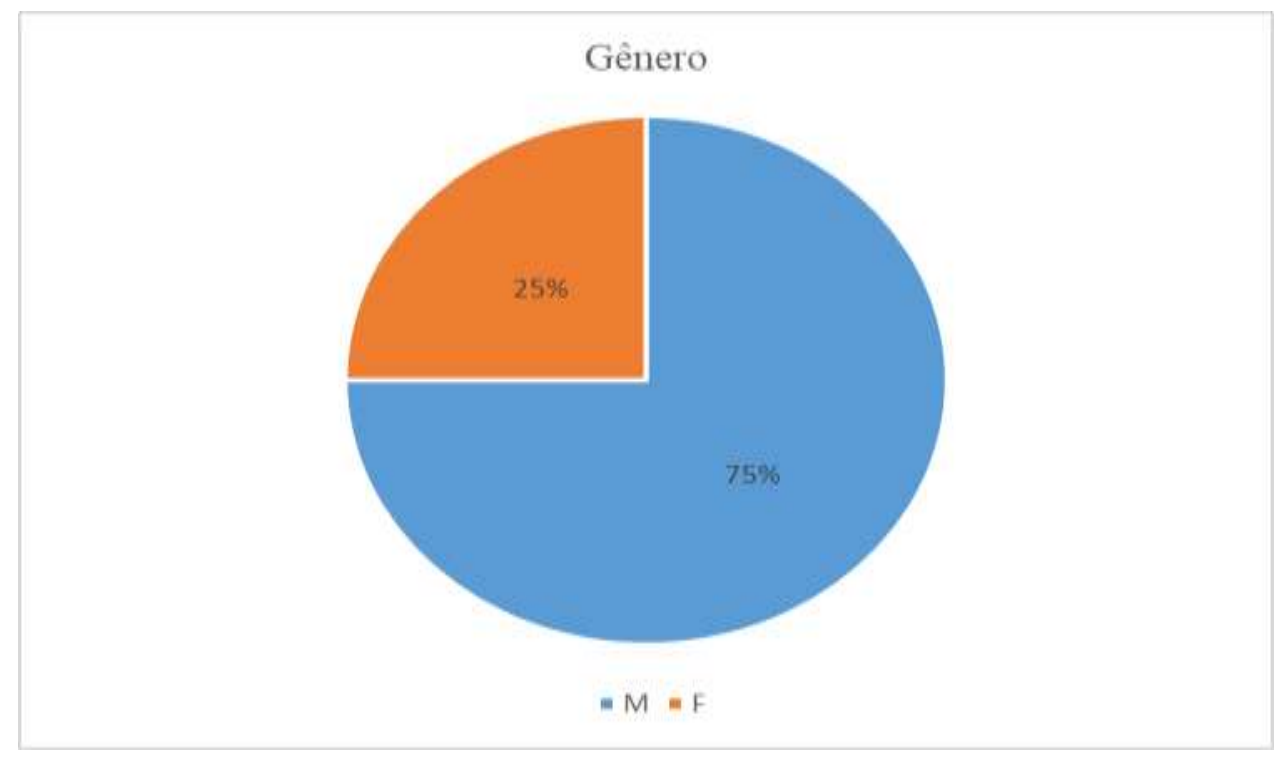

Fonte: Dados da pesquisa. 
O Gráfico 3 mostra a distribuição dos pacientes que tiveram inserção inadequada do dreno torácico, sendo importante ressaltar a maior prevalência do sexo masculino com cerca de $75 \%$ ( $\mathrm{n}=18$ ), quando comparado com o sexo feminino em menor incidência com $25 \%(\mathrm{n}=6)$.

Dentre os 24 pacientes do presente estudo tiveram a confirmação da inserção inadequada do dreno torácico através do exame de tomografia computadorizada de tórax, a principal indicação foi a presença de efusão pleural.

Gráfico 4. Causas da drenagem torácica dos pacientes com inserção inadequada do dreno.

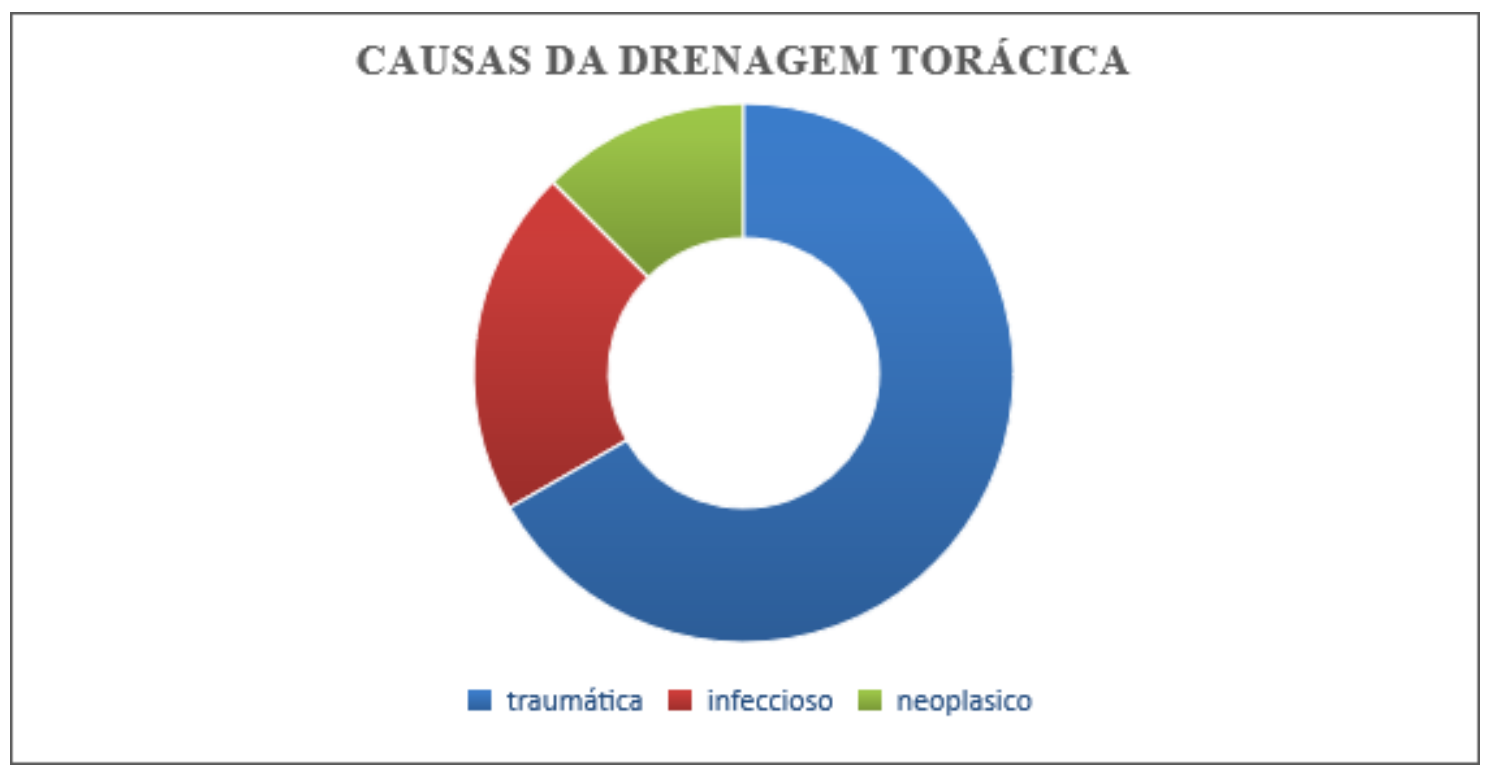

Fonte: Dados da pesquisa.

No Gráfico 4, observa-se uma prevalência de 67\% (n=16) das indicações de drenagem torácica para o manejo de ocorrências traumáticas. A segunda maior prevalência de indicações é por processos infecciosos $21 \%(\mathrm{n}=5)$, o qual dentre elas se destacam a ocorrência empiema pleural, que seria a formações de coleções purulentas com micro-organismos no espaço pleural. Houve uma prevalência de drenagens por conta de processos neoplásicos, o qual conta com $12 \%$ (n=3) das indicações. 
Research, Society and Development, v. 10, n. 4, e27410414135, 2021

(CC BY 4.0) | ISSN 2525-3409 | DOI: http://dx.doi.org/10.33448/rsd-v10i4.14135

\section{Aspectos na imagem}

Figura 1. TC de tórax (janela de mediastino), reconstrução multiplanar oblíqua e plano coronal. Paciente de com suspeita de hemotórax e dreno não funcionante. Exemplo de dreno torácico intra-abdominal inserido pelo hemitórax esquerdo e alcançando o parênquima hepático.
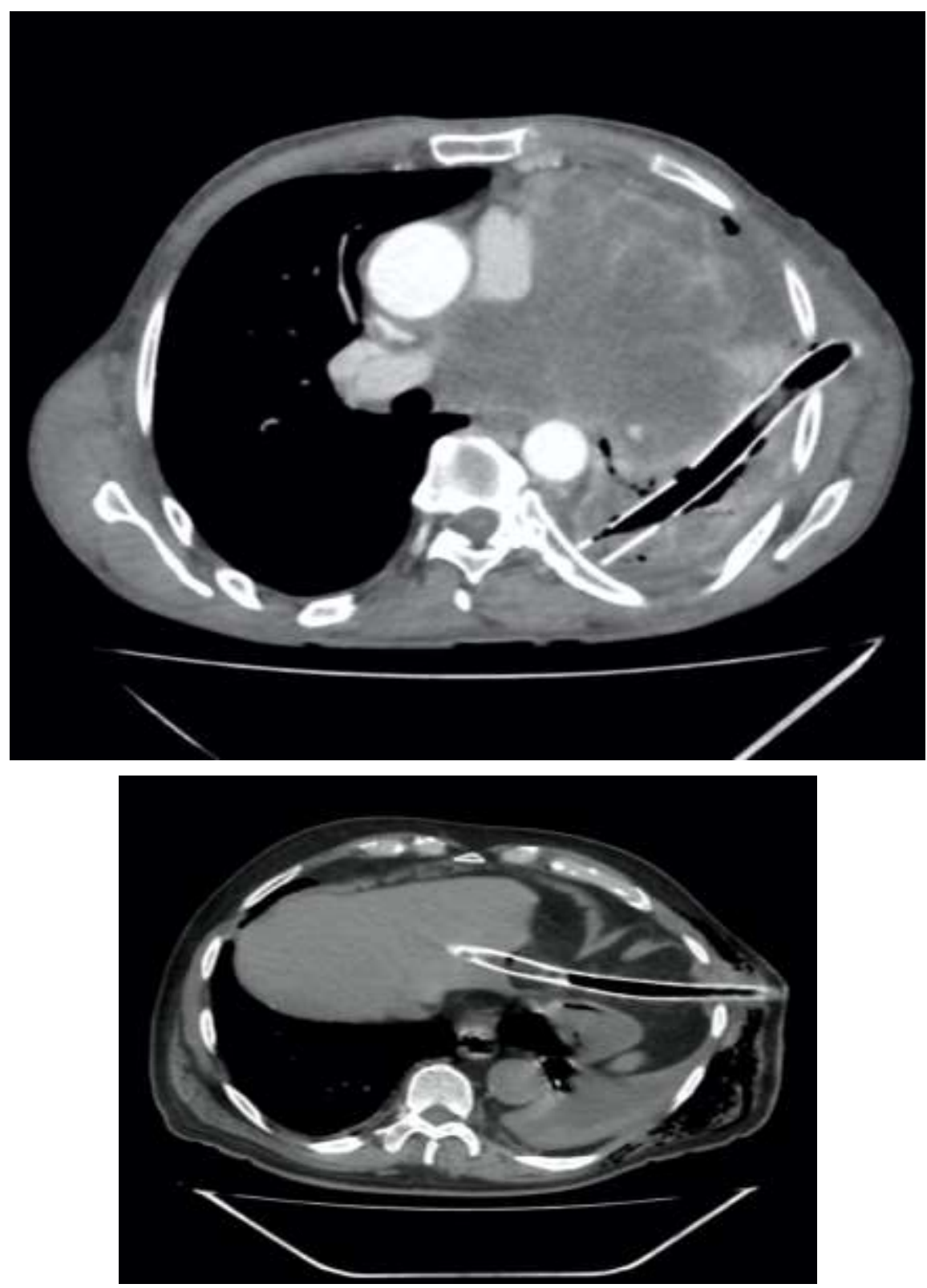

Fonte: Autores. 
Figura 2: TC de tórax (janela de mediastino), reconstrução multiplanar oblíqua em paciente com neoplasia pulmonar. Dreno torácico inserido no interior parênquima pulmonar, adjacente à lesão neoplásica.

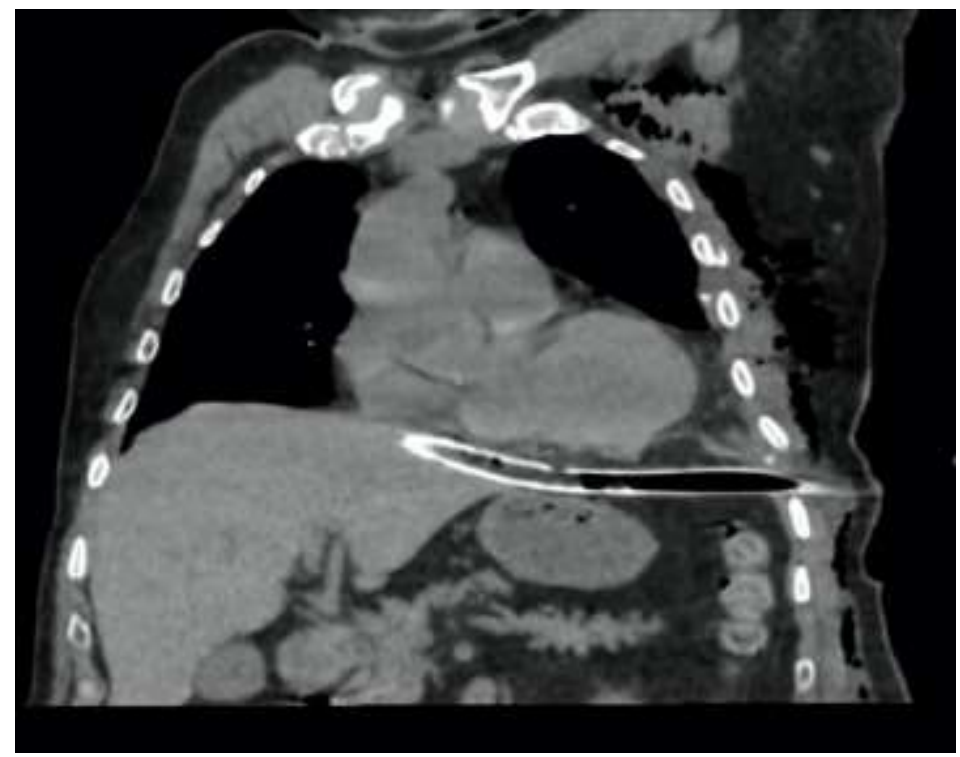

Fonte: Autores.

Figura 3. TC de tórax (janela de mediastino), plano axial. Paciente com neoplasia pulmonar e dreno torácico. Dreno inserido no interior parênquima pulmonar e apresentando acotovelamento no nível da neoplasia.

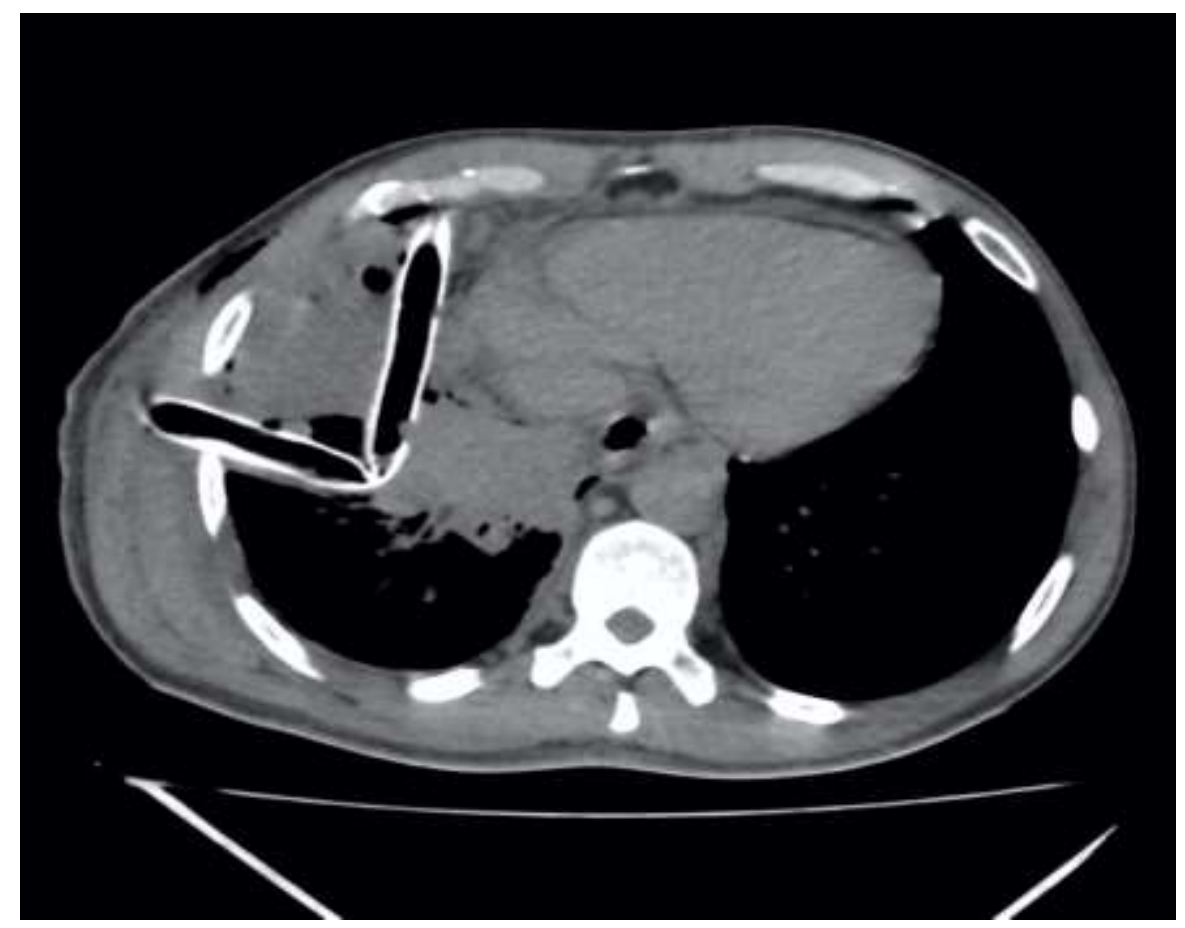

Fonte: Autores. 
Figura 4. TC de tórax (janela de mediastino), reconstrução multiplanar oblíqua. Paciente com ferimento por arma de fogo e dreno torácico. Dreno com inserção subdiafragmática (seta branca), observando-se artefatos metálicos pelo ferimento por arma de fogo (seta vermelha).

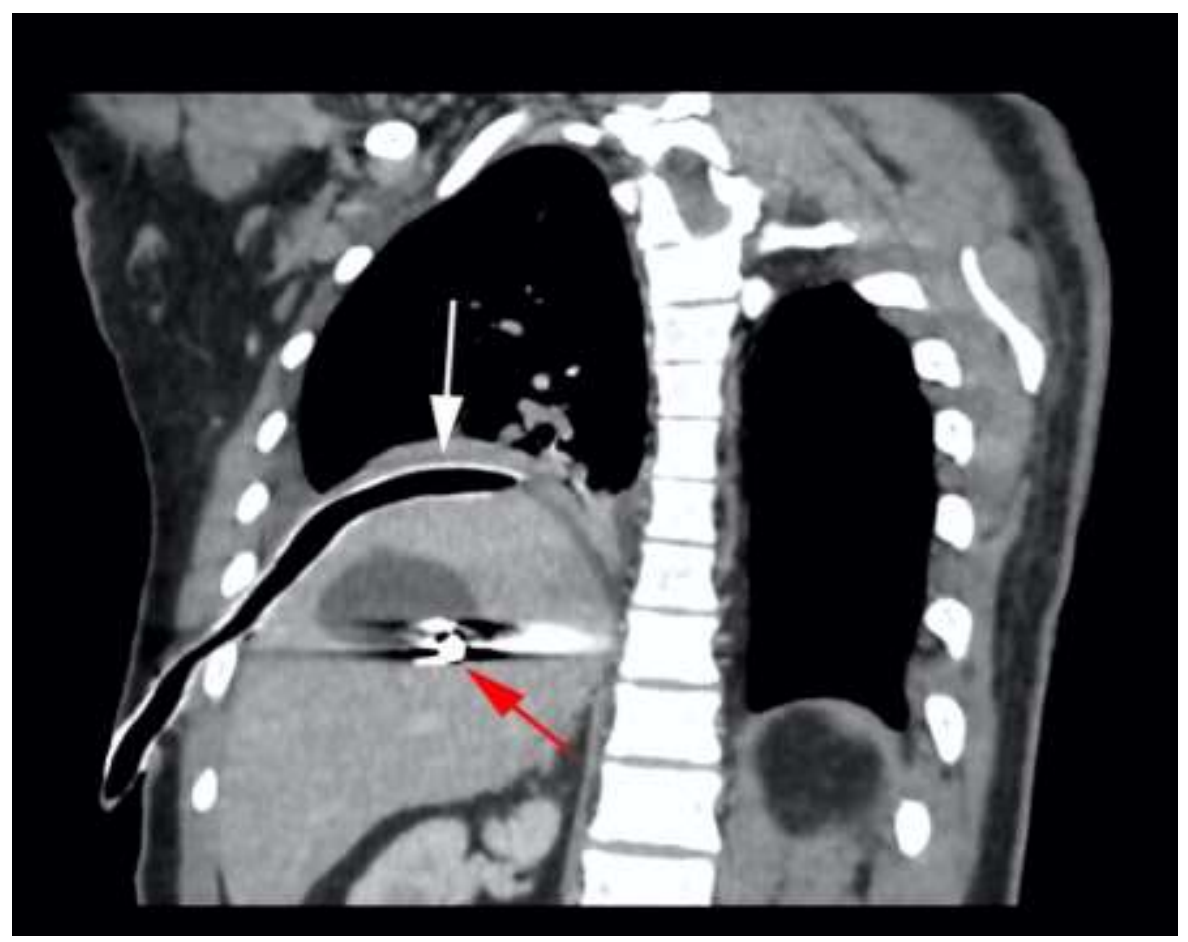

Fonte: Autores.

Figura 5. TC de tórax (janela de parênquima), reconstrução multiplanar oblíqua. Dreno torácico com posicionamento inadequado, observando-se pneumomediastino (seta vermelha) e extenso enfisema subcutâneo (seta azul).

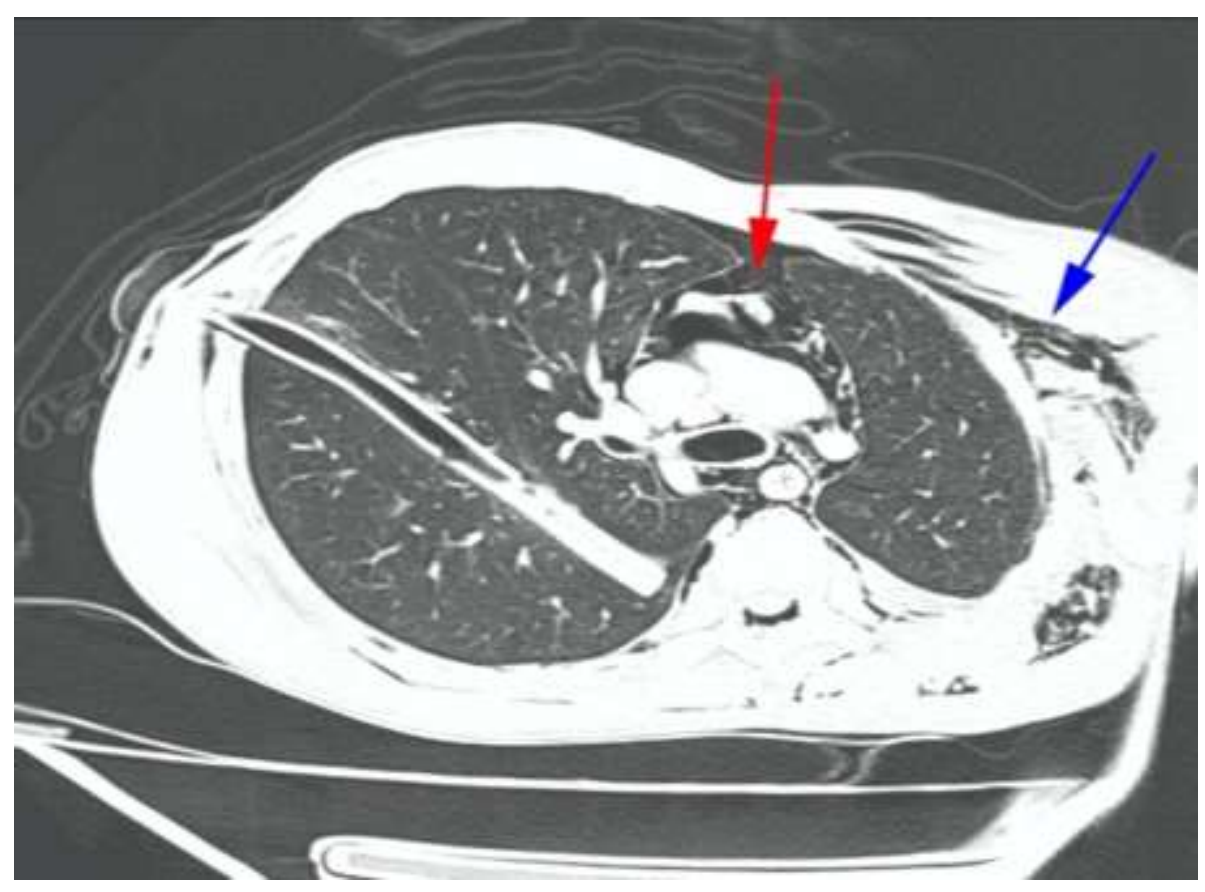

Fonte: Autores. 
Figura 6. TC de tórax (janela de parênquima), reconstrução multiplanar oblíqua. Dreno torácico com posicionamento inadequado, inserido em escavação pulmonar com provável fístula broncopleural associada.
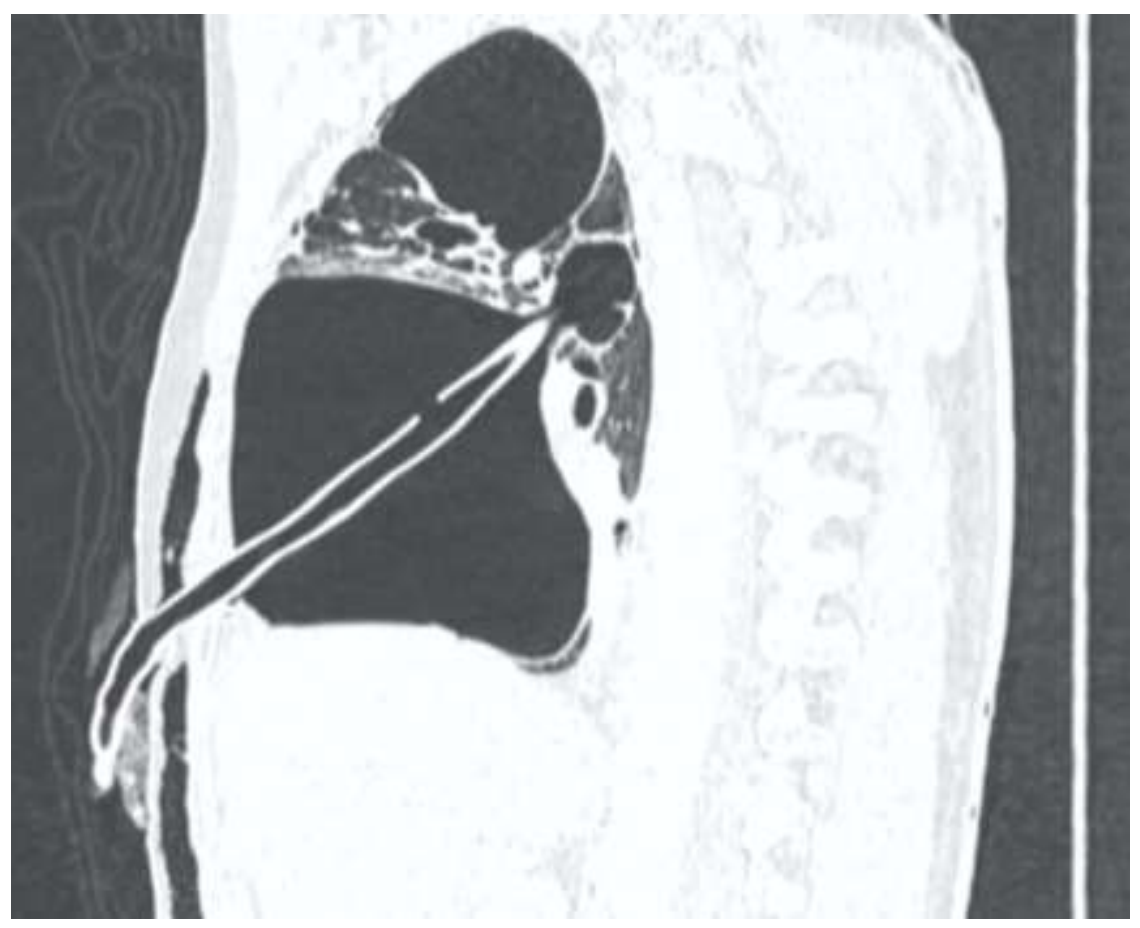

Fonte: Autores.

Figura 7. TC de tórax (janela de mediastino), reconstrução multiplanar oblíqua em paciente com pneumonia. Dreno torácico mal posicionado, inserido no interior parênquima pulmonar e apresentando acotovelamento.

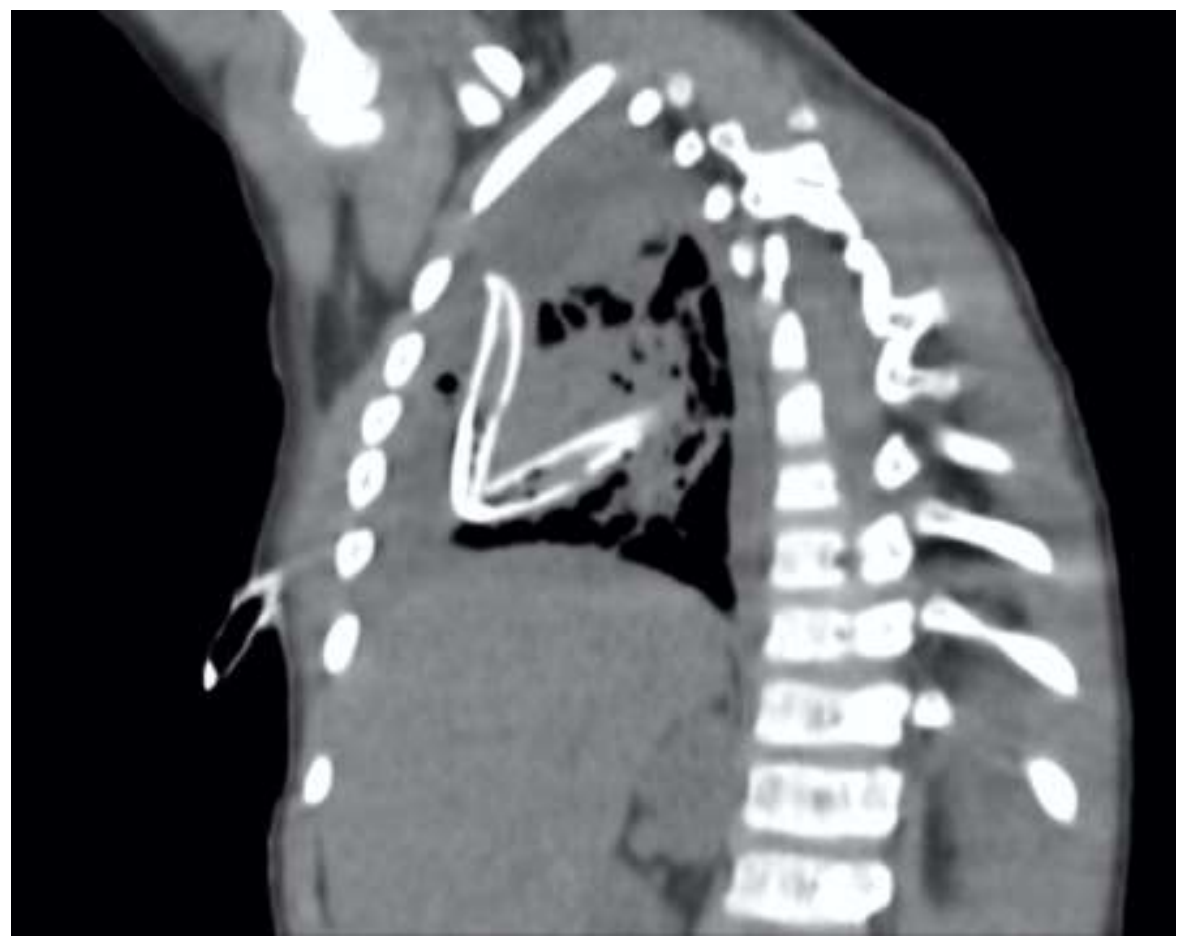

Fonte: Autores. 


\section{Discussão}

A drenagem torácica está entre os procedimentos mais realizados na prática clínica. Tem várias funções importantes na terapêutica como no manejo do pneumotórax, traumas penetrantes, hemotórax, quilotórax, derrames pleurais, fistula broncopleural, pleurodese para condições malignas e benignas, no uso pós-operatório em cirurgia torácica, cardíaca e empiema. Apesar das diversas indicações da técnica, o procedimento tem potencial de morbidade e mortalidade (Filosso, 2017).

Observando o Gráfico 1, percebe-se que ocorreu um aumento considerável de drenagens torácicas com colocação do inadequado do dreno entre os anos 2018 a 2019 no município de Marabá de cerca de 37,5\%. Estudos relacionam a alta incidência de erros pela falta de capacitação prática entre os profissionais da área da saúde (JUNIOR, 2017). Ressalta-se que o no ano de 2018 houve um número menor de pacientes encaminhados do Hospital Municipal de Marabá (HMM), o que também explica essa menor incidência.

Além disso, embora a drenagem torácica seja considerada um procedimento rotineiro, os cuidados com os drenos torácicos variam de acordo com as instituições, e entre as equipes médicas, de fisioterapia e de enfermagem. Essa falta de padronização pode comprometer o resultado e ocasionar complicações. Também é importante ressaltar que a recolocação de dreno após uma inserção inadequada aumenta a chance de infecção, somando-se ao fato de que drenagens realizadas por médicos recém-formados ou residentes tem maior chance de falha técnica (Junior, 2017).

Os pacientes entre 40-59 anos foram prevalentes na inserção inadequada de dreno torácico como mostrado no Gráfico 2 com cerca de $37 \%$ (n=9), seja por conta de um trauma penetrante ou contuso, esses pacientes tinham uma maior incidência de empiema, visto que a idade é um fator que contribui, devido a uma menor reserva fisiológica seja ela cardiovascular ou respiratória, além da presença de doenças crônicas associadas que aumentam a propensão de reter secreções pulmonares, tendo dessa forma uma das principais complicações nessa faixa etária (Neto, 2015).

Em relação a faixa etária e gênero dos pacientes do estudo, nota-se a predominância de pacientes mais jovens e do sexo masculino (Gráfico 3). Esse resultado foi semelhante ao encontrado por Júnior.et.al (2017), em seu estudo no qual houve predomínio do sexo masculino (84,7\%), com média global de idade de 38,2 anos. Na pesquisa esse achado está diretamente relacionado com uma maior incidência de traumas fechados causados por acidentes automobilísticos entre homens jovens.

No presente estudo, observou-se uma prevalência de 67\% $(\mathrm{n}=16)$ das indicações de drenagem torácica provenientes de ocorrências traumáticas como evidenciado no Gráfico 4. Isso corrobora com os resultados obtidos na pesquisa observacional realizada por Morais et al (2016), na qual também houve predomínio da causa traumática na indicação da drenagem torácica, totalizando cerca de $72 \%$ dos pacientes atendidos.

A segunda maior causa de indicação encontrada na pesquisa é por processos infecciosos $21 \%(\mathrm{n}=5)$ evidenciada a partir do Gráfico 4, o qual dentre elas se destacam a ocorrência empiema pleural que seria a formações de coleções purulentas no espaço pleural por processo infeccioso. Em menor prevalência de drenagem é por conta de neoplasia pleural que conta com $12 \%(n=3)$ das indicações. Houve resultado semelhante no perfil epidemiológico traçado por Kogien e Teixeira (2011), no qual a segunda maior indicação clínica foi a presença de empiema pleural (42\%) e a menor foi pela suspeita ou presença de neoplasia pulmonar (10\%).

No presente estudo, a maioria dos achados sobre posicionamento inadequado do dreno foi em localização intraparenquimatosa como mostrado nas Figuras 2 e 5, pois o tubo intraparenquimatoso pode lacerar o tecido, causando pneumotórax, sangramentos, e se prologando pode determina o surgimento de fístulas broncopleurais, bem como infecções com desenvolvimento de abscesso pulmonar. Para evitar tais ocorrências, deve ser solicitado um exame radiológico prévio à inserção do dreno. Nos casos em que houver hemorragias não controladas deve-se considerar toracotomia reparadora imediata (Filosso, 2017). 
Uma complicação incomum, mas que também pode ocorrer após a inserção do dreno é o acotovelamento presentes nas Figuras 3 e 7, onde a migração ou inserção inadequada do tubo ocasiona sua dobra. Na maioria das vezes, não há repercussões clínicas, no entanto, em alguns casos pode acontecer a oclusão do sistema de drenagem, o que diminui a eficácia do procedimento. Nessas situações, o indicado é a retirada do dreno torácico. (Sucena et al, 2010).

Algumas comorbidades dos pacientes podem facilitar a ocorrência do posicionamento inadequado do dreno, como complacência pulmonar diminuída, consolidação do parênquima subjacente ou aderências pleurais significativas. Essas condições impedem o deslocamento normal do pulmão e pioram após a inserção do dreno. Além, disso podem facilitar a laceração pulmonar tardia principalmente com a permanência prolongado do dreno de tórax. A remoção do tubo tem sido indicada como terapia inicial nessas ocorrências (Kwiatt, 2014).

A colocação inadequada de dreno torácico pode resultar em inserção intra-abdominal como apresentada na Figura 1, essa incidência é de difícil quantificação, pois a maioria das informações de lesões intra-abdominais são baseadas em relatos de casos clínicos individuais. A literatura indica que pacientes obesos e possíveis intervenções cirúrgicas anteriores podem resultar em elevação diafragmática e facilitar a inserção na região anatômica incorreta (Filosso, 2017).

Outro achado de imagem encontrado nas análises das tomográficas foi a inserção do tubo no tecido hepático (Figura 1). Sangramentos pelo dreno e instabilidade hemodinâmica grave após drenagem pleural do lado direito deve levantar suspeitas de uma possível lesão nessa região. Lira (2020), comenta que esse é um relato incomum na literatura, ocorrendo principalmente em situações de emergência sem acessos a alguma forma de avaliação por imagem prévia. Outros órgãos que podem ser lacerados pelo tubo torácico incluem estômago, intestinos, baço e rins.

Em alguns casos, o dreno torácico é colocado fora do espaço pleural, especificamente no tecido subcutâneo ou interior dos planos musculares. Este erro está relacionado também pela falta de experiência do profissional e à colocação do dreno em situações de emergências. Para diagnosticar rapidamente esse erro é importante realizar uma radiografia logo após o procedimento e confirmar a posição. Atenção redobrada aos pacientes obesos ou com múltiplas faturas de costelas, o que pode facilitar a colocação extratorácica do dreno (Filosso, 2017).

Outras entidades clínicas que a posição incorreta de um dreno torácico pode causar são o enfisema subcutâneo e pneumomediastino (Figura 5). Essa é uma complicação relativamente comum na sequência de técnicas invasivas ou alguns procedimentos cirúrgicos, incluindo a drenagem torácica. Essa complicação é proveniente da ruptura de alvéolos no local do dreno mal posicionado, que extravasa o ar que progride até as bainhas perivasculares, até alcançar o mediastino. Posteriormente, o ar passa para o tecido adiposo causando o enfisema subcutâneo (Sucena et al, 2010).

Os pacientes que são submetidos a drenagem torácica com dreno mal posicionado após 72 horas de retirada do dreno podem ter a formação de um hemotórax residual, o qual geralmente é diagnosticado por radiografia, no qual é identificado velamentos pleurais quando o volume retido no espaço pleural é de $300 \mathrm{ml}$. Essa complicação além de aumentar o tempo de internação dos pacientes nos hospitais e gerar gastos, é uma das causas importantes de morbidade, visto que o hemotórax residual contribui com cerca de 12 a 16\% para a ocorrência de empiema pleural (Neto, 2012).

A maioria das pesquisas relaciona o aumento de complicações ligadas com o mal posicionamento do dreno torácico com a falta de investimento em cursos de capacitação para os profissionais da área de saúde e de padronização de protocolos. O indicado seria ampliar a oferta de métodos de imagem rápidos e de fácil acesso, dentre elas a utilização de ultrassom que pudesse guiar o procedimento de drenagem do tórax e dessa forma localizar exatamente o local a ser drenado sem o comprometimento de estruturas adjacentes. (Menegozzo, 2018).

Além disso, prática contínua da técnica de drenagem torácica gera uma maior habilidade e principalmente segurança para a sua realização correta. O investimento em treinamento desde a graduação, como a elaboração de simuladores de baixo 
custo para que os estudantes de medicina principalmente os internos pudessem ter mais domínio da técnica, poderia reduzir drasticamente as lesões iatrogênicas após uma inadequada inserção de dreno torácico (Bettega, 2011).

Ademais, a falta de gerenciamento dos drenos torácicos também está como uma das causas que contribui para as complicações do procedimento, isso porque a falta de verificação de oscilação do selo d'água é um dos sinais mais negligenciado. É um dos indicativos que o dreno estaria mal colocado e com isso acaba por prejudicar diretamente a correta ventilação do paciente aumentando dessa forma consideravelmente a morbimortalidade. Por isso, a elaboração de um protocolo que padronizem o gerenciamento da drenagem torácica é benéfico para reduzir os agravantes ocasionados por este (Morais, 2016).

\section{Considerações Finais}

Com essa pesquisa, identificamos o grupo que tem mais incidência de complicações relacionadas ao posicionamento inadequado do dreno é o sexo masculino (75\%) entre 40 a 59 anos (37,5\%) sendo que a drenagem foi indicada por causas traumáticas $(67 \%)$. Além disso o local mais comum de inserção do dreno incorreto ocorreu na região intraparenquimatosa pulmonar, causando como principais complicações o pneumotórax, sangramentos e nos casos mais avançados o surgimento de fistulas broncopleurais.

Essas evidencias corrobora o importante papel que os exames de imagens têm para auxiliar na prevenção e deteç̧ão dos achados de imagem de lesões iatrogênicas por drenos torácicos mal posicionados, como por exemplo a utilização de ultrassom para evitar a má colocação do dreno torácico e da capacidade técnica do executor deste exame para localizar de forma exata do local a ser drenado.

Nesse sentido, demonstra a necessidade de uma padronização de protocolo para que a ação e manejo a esses pacientes seja realizado de forma rápida e efetiva, a fim de evitar iatrogenias. Além disso é necessário que tenha um engajamento técnico da equipe que estará à frente na realização do procedimento de drenagem torácica para que dessa forma tenha-se um menor número de inserção inadequada do dreno torácico.

\section{Referências}

Abreu, E. M. S. D., Machado, C. J., Neto, M. P., \& Sanches, M. D. (2015). Impacto de um protocolo de cuidados a pacientes com trauma torácico drenado. Rev. Col. Bras. Cir. 42(4), 231-237.

Araújo, P. H. X. N. D., Terra, R. M., Santos, T. D. S., Chate, R. C., Paiva, A. F. L. D., \& Fernandes, P. M. P. (2017). Posicionamento intrapleural, guiado por ultrassonografia, de cateteres pleurais: influência na expansão pulmonar imediata e na pleurodese em pacientes com derrame pleural maligno recorrente. $J$ Bras Pneumol. 43(3), 190-194.

Bettega, A. L et al. (2011). Simulador de dreno de tórax: desenvolvimento de modelo de baixo custo para capacitação de médicos e estudantes de medicina. Rev Col Bras Cir. 46(1)

Cipriano, F. G. \& Dessote, L. U. (2011). Drenagem pleural. Medicina. 44(1), 70-8.

Davies, H. E., Davies, R. J. O. \& Davies, C. W. H. (2010). Management of pleural infection in adults: British Thoracic Society pleural disease guideline. Thorax. 65(2), 41-53

Filosso, P. L et al. (2017). Errors and complications in chest tube placement. Thorac Surg Clin. 27, 57-67.

Huggins, J. T., Carr, S., \& Woodward, G. A. (2019). Placement and management of thoracostomy tubes and catheters in adults and children. Coliins, K. A., ed. UpToDate. Waltham, MA: UptoDate Inc. https://www.uptodate.com/contents/placement-and-management-of-thoracostomy-tubes-and-catheters-in-adultsand-children?

Junior, C. A. B., Botelho, A. B., Linhares, A. D. C., Oliveira, M. S. D., Veronese, G., Junior, C. R. N., Batista, L. C., \& Diogo, M. A. K. (2017). Perfil das pacientes vítimas de trauma torácico submetidos à drenagem de tórax. Rev. Col. Bras. Cir. 44(1), 027-032.

Kwiatt, M., Tarbox, A., Seamon, M. J, et al. (2014). Thoracostomy tubes: A comprehensive review of complications and related topics. Int J Crit Illn Inj Sci. 4(2), 143-155.

Kogien, M., Teixeira, C. A. (2011). Toracotomias: estudo epidemiológico em um hospital de grande porte. Rev enferm UFPE on line. 5(3), 611-617. 
Research, Society and Development, v. 10, n. 4, e27410414135, 2021

(CC BY 4.0) | ISSN 2525-3409 | DOI: http://dx.doi.org/10.33448/rsd-v10i4.14135

Lira, M. (2020). Hospitais regionais já têm planos de enfrentamento à Covid-19. Agência Pará. Março. https://agenciapara.com.br/noticia/18539/.

Lim, K. E., Tai, S. C., Chan, C. Y et al. (2005). Diagnosis of malpositioned chest tubes after emergency tube thoracostomy Is computed tomography more accurate than chest radiograph? Journal of Clinical Imaging. 29, 401-405.

Medeiros, B. J. D. C. (2019). Cuidados Padronizados Com Dreno De Tórax. Aspectos Técnicos e Manejo. Universidade Federal do Amazonas. Manaus, AM.

Menegozzo, C. A. M., Pflug, A. R. M., \& Utiyama, E. M. (2018). Como reduzir complicações relacionadas à drenagem pleural utilizando uma técnica guiada por ultrassom. Rev Col Bras Cir. 45(4).

Mendes, C. A., \& Hirano, E. S. (2018). Fatores preditivos de complicações na drenagem torácica pós-trauma. Dissertação - Unicamp. Campinas, SP.

Morais, A. C. C., Lemos, M. M., Marques, V. D., \& Vladimir. C. O. P. (2016). Protocolo institucional para padronizar o gerenciamento do sistema de drenagem torácica, da cirurgia à assistência de enfermagem, em um hospital regional do norte do Paraná. Acta Scientiarum. 38(2), 173-177.

Neto, J. B. D. R., Neto, M. P., Hirano, E. S. H., Rizou, S., Junior, B. N., \& Fraga, G. P. (2012). Abordagem do hemotórax residual após a drenagem torácica no trauma. Rev. Col. Bras. Cir. 39(4), 344-349.

Neto, M. P., Resende, V., Machado, C. J., Abreu, E. M. S. D., Neto, J. B. B., \& Sanches, M. D. (2015). Fatores associados ao empiema em pacientes com hemotórax retido pós-traumático. Rev. Col. Bras. Cir. 42(4), 224-230.

Perazzo, A, Gatto, P., Barlascini, C., Bravo, M. F., \& Nicolini. A. (2013). A ultrassonografia pode reduzir o risco de pneumotórax após toracocentese? J Bras Pneumol. 40(1), 6-12.

Sucena, Maria et al. (2010). Enfisema subcutâneo maciço - Tratamento com drenos subcutâneos. Rev Port Pneumol, Lisboa. 16(2), 321-329. 